\title{
MEASUREMENT of NON-LINEARITIES USING SPECTRUM ANALYSIS of DRIVEN BETATRON OSCILLATION *
}

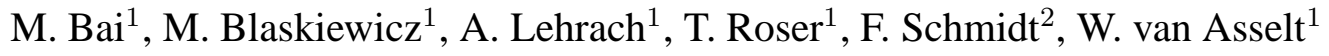 \\ ${ }^{1}$ Brookhaven National Laboratory, Upton, NY 11973, U.S.A \\ ${ }^{2}$ CERN, Geneva 23, CH
}

\begin{abstract}
Resonance driving terms can be derived from the frequency analysis of turn-by-turn betatron oscillation data. This paper demonstrates that the same information can also be drawn from the spectral analysis of a driven oscillation adiabatically excited by an $\mathrm{rf}$ dipole. The advantage of this method is that a large betatron oscillation amplitude can be sustained without loosing the coherence signal. The frequency spectrum of the driven oscillation is composed of multiples of the rf dipole modulation frequency which can be interpreted as resonance driving terms. This analysis has been applied to the data taken at the Brookhaven AGS. The adiabatically excited coherent oscillation is also very useful in measuring the betatron tune parasitically. The data taken during the AGS high intensity proton program is also presented.
\end{abstract}

\section{INTRODUCTION}

With non-linearities in an accelerator, the Fourier spectrum of action-angle variables of the turn by turn beam position data contains not only the primary line at betatron tune but also the spectral lines at its harmonics [?]. By analyzing the spectral lines at the harmonics of the betatron tune, one can then extract the driving terms of the high order resonances [?]. Table 1 lists the corresponding spectral lines on the Fourier spectrum for the 3rd and 4th order of resonances.

Table 1: high order resonances and the corresponding spectral lines

\begin{tabular}{|c|c|c|}
\hline $\begin{array}{c}\text { order of } \\
\text { resonance }\end{array}$ & $\begin{array}{c}\text { spectral line } \\
\text { harmonics }\end{array}$ & $\begin{array}{c}\text { driving } \\
\text { source }\end{array}$ \\
\hline 3 & 2 & sextupole \\
\hline 4 & 3 & octupole \\
\hline
\end{tabular}

To get a decent measurement by using this method, turn by turn beam position data of a long lasting coherent oscillation is required. However, due to the tune spread in the beam, the coherent oscillation induced by a one-turn kicker normally gets decohered pretty quickly. The alternative way is to drive beam with an oscillating dipole field (ac dipole) oscillating with a frequency at the vicinity of the

\footnotetext{
* The work was performed under the auspices of the US Department of Energy
}

intrinsic betatron frequency. This method has been demonstrated in the Brookhaven AGS. By operating an rf dipole in an adiabatic fashion, namely slowly energizing from zero field to the desired field strength, a long-lasting coherent oscillation can excited without increasing the beam emittance after the rf dipole is adiabatically de-energized [3].

Unlike the free betatron oscillation spectrum, the primary spectral line in the spectrum of the driven coherent betatron oscillation excited by an ac dipole is the driving frequency, and the amplitude of the higher harmonics of the driving tune is proportional to the high order resonance driving terms.

In addition to the non-linearities measurement, one can also use an ac dipole to measure betatron tune by driving the beam with two frequencies simultaneously. For a linear machine, the amplitude of the excited coherent betatron oscillation $Z_{c o h}$ is given by

$$
Z_{c o h}=\frac{\Delta B_{m} L}{4 \pi \delta B \rho} \beta_{z},
$$

where $\Delta B_{m}$ is the ac dipole field oscillating amplitude, $L$ is the ac dipole length and $\beta_{z}$ is the betatron amplitude function at where the ac dipole is. $B \rho$ is the rigidity of the beam and $\delta=\left|\nu_{m}-\nu_{z}\right|$ is the distance between the ac dipole tune $\nu_{m}=\frac{f_{m}}{f_{0}}$ and the betatron tune $\nu_{z}$. Here, $f_{m}$ is the ac dipole frequency and $f_{0}$ is the beam revolution frequency.

According to Eq. 1, the coherence amplitude $Z_{1}$ with ac dipole tune $\nu_{1}$ and the coherence amplitude $Z_{2}$ with ac dipole tune $\nu_{2}$ have the following relation

$$
Z_{1}\left(\nu_{z}-\nu_{1}\right)=Z_{2}\left(\nu_{2}-\nu_{z}\right)
$$

assuming the oscillating field amplitude is the same for the two ac dipole tunes and $\nu_{1} \leq \nu_{z} \leq \nu_{2}$. Therefore, we have

$$
\nu_{z}=\frac{Z_{1} \nu_{1}+Z_{2} \nu_{2}}{Z_{1}+Z_{2}} .
$$

\section{EXPERIMENTAL RESULTS AT THE BROOKHAVEN AGS}

\subsection{Measure the driving term of high order res- onance}

During the last year RHIC polarized proton experiment, we explored the possibility of using the adiabatically excited coherent oscillation to measure the high order resonance terms in the AGS. A horizontal rf dipole was used to adiabatically excite a coherent betatron oscillation at the 


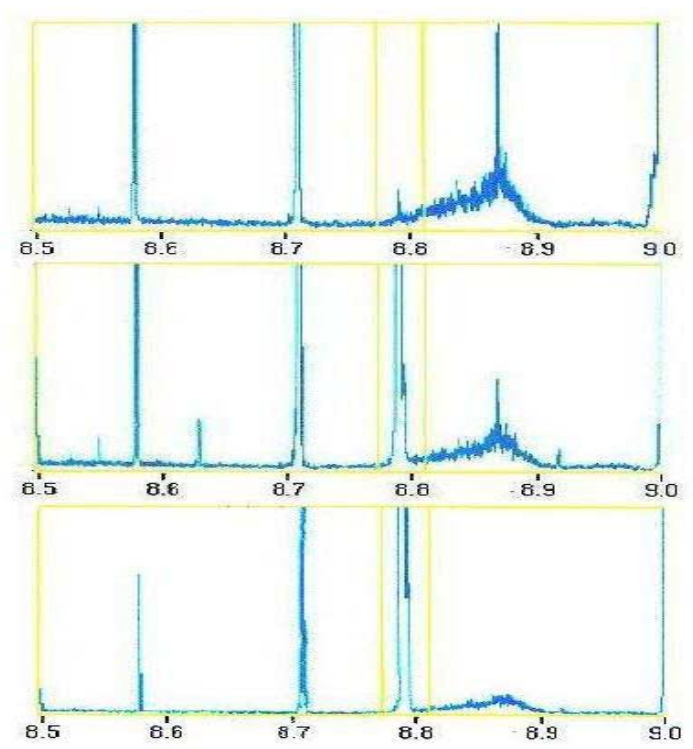

Figure 1: Frequency spectrum of the coherent betatron oscillation for different octupole settings. The horizontal axis is the tune and the vertical axis is the amplitude of the spectral lines with arbitrary unit. From top to bottom, the three plots correspond to the octupole strength of gain $-12,12$ and 0 , respectively.

AGS extraction energy. We also powered the AGS octupoles to different strength and measured the corresponding frequency spectrums of the coherent betatron oscillation as shown in Figure 1. The 3rd harmonic line of the rf dipole tune of 0.71 with the octupole strength of gain -12 is much stronger than the one with the octupole strength of gain 12 as shown in the top and middle plots, respectively. This harmonic line disappears when the octupole is turned off as demonstrated in the plot at the bottom.

A series of turn by turn beam position data with different coherent oscillation amplitude were also recorded through a digital oscilloscope. The Fourier spectrum of the actionangle of the turn by turn bpm data was calculated by using the SUSSIX program $[2,4]$. Figure 2 shows the amplitude of the 2nd harmonic line of the driving frequency normalized by the primary line amplitude as a function of different betatron oscillation size. For this measurement, the AGS octupoles were turned off and two families of sextupoles were used to minimize the chromaticity. However, the dependence of the 2nd harmonic spectral line amplitude on the coherent oscillation does not fit well with the quadratic behavior of the 3rd order resonance. This is because the oscilloscope which was used to digitize the analog turn by turn beam position signal was not properly set up which greatly degrade the quality of the beam position data.

\subsection{Measure betatron tune using an ac dipole}

To verify the method of using driven oscillations to measure the betatron tune, we resonated the vertical rf dipole with two tunes of $\nu_{1}$ and $\nu_{2}$ at the same time. The oscillat-

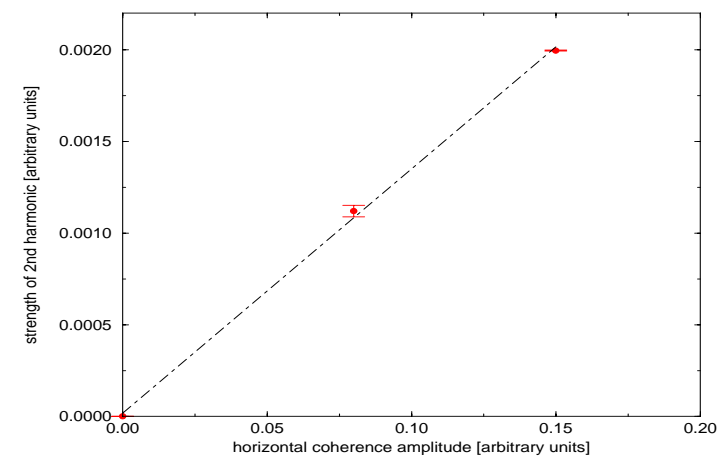

Figure 2: Measured 2nd harmonic spectral line amplitude as a function of the size of the horizontal oscillation.
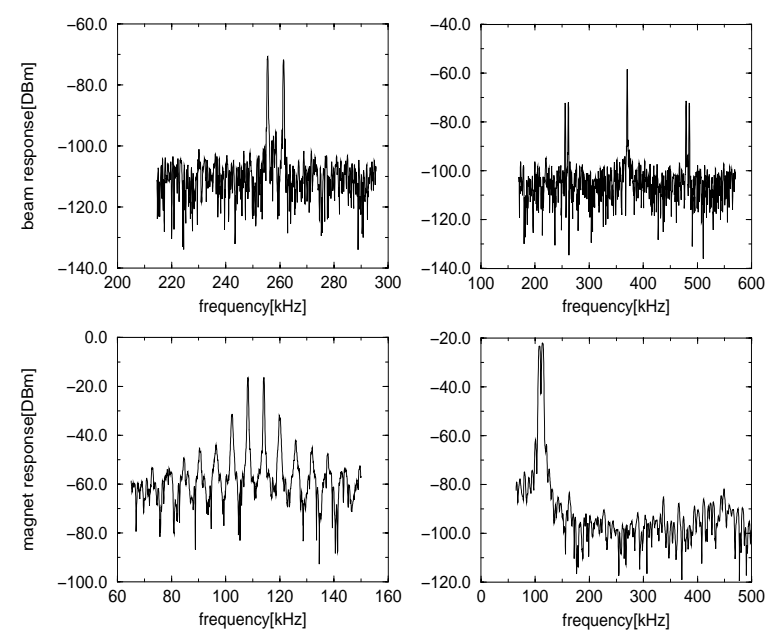

Figure 3: The top right plot is the frequency spectrum of the beam position signal. The center line around $370 \mathrm{kHz}$ is the beam revolution frequency and the two pairs of the spectral lines on either side of the revolution line correspond to the two driving frequencies of the rf dipole. The top left plot is the blow-up of the pair of the lines at the left side of the revolution line. The plot at the lower right corner is the spectrum of the vertical rf dipole current signal, and the plot at its left side is the zoom in the range between $70 \mathrm{kHz}$ and $150 \mathrm{kHz}$. Since the rf dipole field at the two driving frequencies are about equally strong, the amplitudes of the driven coherent oscillation at the two frequencies are very close.

ing dipole field is given by

$$
B(\theta)=B_{m a} \cos \left(\nu_{1} \theta\right)+B_{m b} \cos \left(\nu_{2} \theta\right),
$$

where $\theta$ is the azimuthal angle along the accelerator. The turn by turn beam position signal was analyzed by a HP spectrum analyzer. Figure 3 shows a typical measured spectrum of the coherent oscillation.

The turn by turn beam position spectrum were measured for different vertical betatron tune settings. Figure 4 shows the calculated tunes using Eq. 3 for different vertical betatron tune settings. It is clear that the tunes calculated from Eq. 3 follow the vertical betatron tunes very well. 

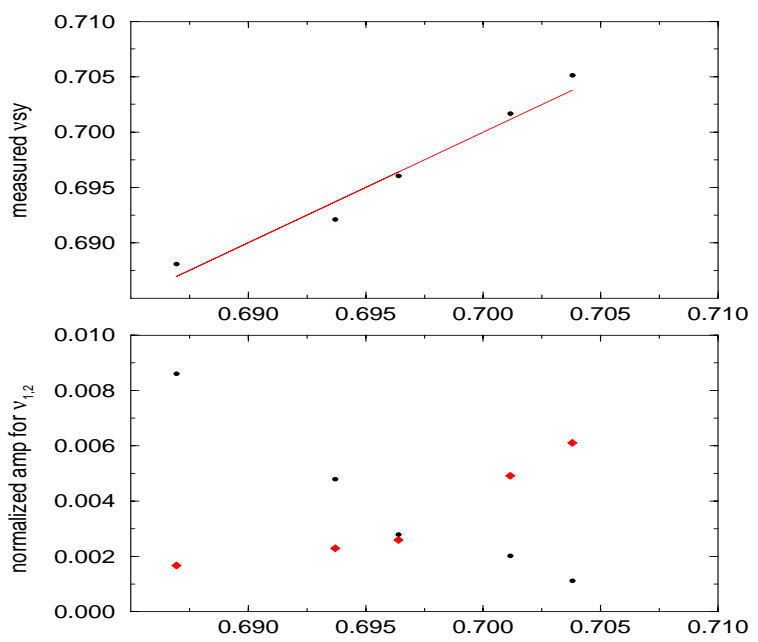

Figure 4: The horizontal axis of the top plot is the vertical betatron tune measured by pulse kicking the beam. The top plot shows that the tunes calculated from Eq. 3 agree well with the vertical betatron tunes we measured. The plot at the bottom is the amplitude of the spectral lines of the bpm spectrum at the two driving tunes $\nu_{1}$ and $\nu_{2}$. The amplitude is normalized by the corresponding spectral line amplitude of the rf dipole current Fourier spectrum.

\section{CONCLUSION}

We are very encouraged by the results of the parasitic experiment at the Brookhaven AGS of exploring the possibility of measuring the non-linear resonance terms by analyzing the Fourier spectrum of a coherent oscillation driven by an ac dipole. To fully demonstrate the feasibility of this method, we plan to perform more studies during the RHIC 2001 run. The advantage of using a driven oscillation is that the coherence of the oscillation can be well maintained for a long time. In addition to the non-linearity measurement, we have also demonstrated that one can measure the betatron tune by using an ac dipole to drive the beam with two driving frequencies nearby the betatron oscillation frequency.

\section{ACKNOWLEDGEMENT}

The authors would like to thank Dr. P. Cameron for providing us the HP spectrum analyzer. The authors also would like to thank K. Zeno for the help on setting up the octupoles.

\section{REFERENCES}

[1] R. Bartolini and F. Schmidt, "Normal Form via Tracking or Beam Data”, Part. Accel. 59, pp. 93-106, (1998)

[2] F. Schmidt, "Measurement of Resonance Driving Terms at the CERN SPS using turn-by-turn data from beam position monitors", this conference.

[3] M. Bai et al., Physical Review E, 5(1997).
[4] R. Bartolini and F. Schmidt, "SUSSIX: A Computer Code for Frequency Analysis of Non-Linear Betatron Montion", CERN SL/Note 98-017 (AP), 1999. 